\title{
Postmarketing surveillance of the safety profile of infliximab in 5000 Japanese patients with rheumatoid arthritis
}

\author{
T Takeuchi, ${ }^{1}, 14$ Y Tatsuki, ${ }^{2}$ Y Nogami, ${ }^{3}$ N Ishiguro, ${ }^{1,14}$ Y Tanaka, ${ }^{5,14}$ H Yamanaka, ${ }^{6,14}$ \\ N Kamatani, ${ }^{6,14}$ M Harigai, ${ }^{7,14} \mathrm{~J} \mathrm{Ryu},{ }^{8,14} \mathrm{~K}$ Inoue, ${ }^{9,14} \mathrm{H}$ Kondo, ${ }^{10,14} \mathrm{~S}$ Inokuma, ${ }^{11,14}$ \\ T Ochi, ${ }^{12,14}$ T Koike ${ }^{13,14}$
}

${ }^{1}$ Division of Rheumatology and Clinical Immunology, Saitama Medical Center, Saitama Medical University, Saitama, Japan; ${ }^{2}$ Pharmaceuticals Sales \& Marketing Headquarters, Tanabe Seiyaku Co., Ltd, Osaka, Japan; ${ }^{3}$ Compliance $\&$ Assurance Headquarters, Tanabe Seiyaku Co., Ltd, Osaka, Japan; ${ }^{4}$ Department of Orthopaedic Surgery, Nagoya University School of Medicine Aichi, Japan; ${ }^{5}$ The First Department of Internal Medicine, University of Occupational \& Environmental Health, School of Medicine, Fukuoka, Japan; ${ }^{6}$ Institute of Rheumatology, Tokyo Women's Medical University, Tokyo,

Japan; ' Department of

Pharmacovigilance and Department of Medicine and Rheumatology, Tokyo Medical and Dental University, Tokyo, Japan; ${ }^{8}$ Department of Orthopaedic Surgery, Nihon University School of Medicine, Tokyo, Japan; ${ }^{9}$ Department of Orthopaedic Surgery, Tokyo Women's Medical University, Medical Center East, Tokyo, Japan; ${ }^{10}$ Kitasato Institute Medical Center Hospital

Saitama, Japan; ${ }^{11}$ Departmen of Allergy and Immunological Diseases, Tokyo Metropolitan Komagome Hospital, Tokyo, Japan; ${ }^{12}$ National Hospital Organization Sagamihara National Hospital, Kanagawa, Japan; ${ }^{13}$ Department of Medicine II, Hokkaido University Graduate School of Medicine, Hokkaido, Japan; ${ }^{14}$ Member of the Committee on PostMarketing Surveillance of Japan College of Rheumatology

Correspondence to: Tsutomu Takeuchi, Division of Rheumatology and Clinica Immunology, Saitama Medical Center, Saitama Medical University, 1981 Kamoda, Kawagoe, Saitama 350-8550, Japan; tsutake@saitama-med. ac.jp

Accepted 14 July 2007 Published Online First

2 August 2007

\section{ABSTRACT}

Objectives: A large-scale postmarketing surveillance (PMS) study was carried out to determine the safety profile of infliximab in Japanese patients with rheumatoid arthritis (RA).

Methods: The PMS study was performed for all patients with RA who were treated with infliximab. They were consecutively registered in the PMS study at the initiation of infliximab treatment and were prospectively monitored with all adverse events noted for a period of 6 months. All case reports, which include safety-related events, were collected monthly.

Results: Adverse drug reactions (ADRs) were assessed for 6 months in 5000 patients who were consecutively enrolled in the PMS study. The incidence rates of total and serious ADRs were $28.0 \%$ and $6.2 \%$, respectively. "Infections" or "respiratory disorders" were most commonly observed among serious ADRs. Bacterial pneumonia developed in $2.2 \%$, tuberculosis in $0.3 \%$, suspected Pneumocystis jiroveci pneumonia (PCP) in $0.4 \%$ and interstitial pneumonitis in $0.5 \%$. Bacterial pneumonia (for which individuals of male gender, of older age and those with advanced rheumatoid arthritis and comorbid respiratory disease were most at risk) began to develop immediately after the start of treatment, while tuberculosis, PCP and interstitial pneumonitis developed about 1 month later. Serious infusion reactions were observed in $0.5 \%$ and were more likely to occur in patients who had participated in previous clinical trials of infliximab.

Conclusion: This postmarketing surveillance study of patients treated with infliximab showed that infliximab in combination with low-dose MTX was well tolerated in Japanese patients with active RA.

Infliximab, an antihuman TNF- $\alpha$ chimeric monoclonal antibody, has been proven to be efficacious in the treatment of a number of inflammatory diseases (ATTRACT, ASPIRE, ASSERT, IMPACT ACCENT, ACT 1,2, SPIRIT and BeSt). ${ }^{1-9}$ Clinical trials of RA treatments have demonstrated that infliximab not only improves clinical signs and symptoms, but also inhibits joint destruction. Given that TNF is necessary for host defense against infections, one can speculate that the incidence of infection may increase during treatment with TNF inhibitors. Although the incidence of serious infections in patients treated with infliximab plus methotrexate (MTX) has been shown to be comparable with those treated with MTX alone in a number of clinical trials, the incidence of Mycobacterium tuberculosis is reported to be higher in RA patients treated with infliximab in endemic regions, suggesting that its appearance is due to reactivation of latent tuberculosis. ${ }^{10}$ The incidence of tuberculosis in Japan is 24.8/100 000 per year (2003 statistics), which is 5 times greater than that of the US. Thus, the potential risk of tuberculosis is a major concern, considering that TNF inhibitors would be widely used after their approval for the treatment of RA in Japan. The Ministry of Health, Labour and Welfare (MHLW) required that a large-scale postmarketing surveillance (PMS) study be carried out as a condition for the approval of infliximab. This PMS study was developed to fulfil that requirement to monitor all adverse drug reactions (ADRs) in every RA patient treated with infliximab and to add to the literature on the risks and benefits of biologic therapy. This report presents the ADRs observed in the 5000 patients enrolled in the study. Bacterial pneumonia, $P$ jiroveci pneumonia (PCP), interstitial pneumonitis and infusion reactions are also discussed in this report.

\section{METHODS}

The specifics of the infliximab PMS study included a prospective collection of all adverse events (AEs) that all patients beginning infliximab therapy might encounter for a period of 6 months. To ensure complete registration and quality data, the registration and reporting were all centrally performed to maximise completeness in capturing information. Only institutions willing to comply with the protocol had access to infliximab during this investigation. The Committee on PMS under the auspices of the Japan College of Rheumatology (JCR) evaluated data in collaboration with a pharmaceutical company (Tanabe Seiyaku Co. Ltd). The MHLW approved the protocol of the PMS study and instructed the investigators to perform the PMS study according to Good PostMarketing Surveillance Practice, which is the authorised standard for PMS studies of approved drugs in clinical practice; therefore, no formal ethics committee approval was necessary.

\section{Patients}

All patients treated with infliximab between July 2003 and December 2004 were enrolled in the study, and each individual patient was followed up for 6 months. The study ended in June 2005 According to the Japanese guidelines for the use of 
infliximab, ${ }^{11}$ the enrolled patients had to have active disease, defined as having $\geqslant 6$ tender joints, $\geqslant 6$ swollen joints and Creactive protein $\geqslant 2.0 \mathrm{mg} / \mathrm{dL}$ or an erythrocyte sedimentation rate $\geqslant 28 \mathrm{~mm} / \mathrm{h}$, despite treatment with MTX at a dose of more than $6 \mathrm{mg} /$ week for at least 3 months. At the time of enrolment, patients gave informed consent for the treatment.

\section{Protocol design}

All patients were consecutively registered upon initiation of infliximab treatment by documenting the patients' demographics using a central registration method. Upon completion of registration, a registration number was given to the patient that was dependent on the exact time and date of registration; all the patients were prospectively monitored for AEs for 6 months thereafter. Infliximab was infused at $3 \mathrm{mg} / \mathrm{kg}$ body weight at weeks 0,2 and 6 , and then every 8 weeks thereafter, with concomitant use of MTX (maximum approved dose in Japan: $8 \mathrm{mg} /$ week). There was no limitation on the concomitant use of glucocorticosteroid or disease-modifying antirheumatic drugs during the study. Patients were queried as to the incidence of AEs at every visit to the investigators, and the investigators reported all AEs that occurred for 6 months after the start of infliximab treatment (whether or not the treatment was continued) as well as all medications and concomitant drugs to the pharmaceutical company. All case reports were collected monthly by the company.

AEs were recorded with the physician's assessment of causality (infliximab or concomitant drugs), severity according to the International Conference on Harmonization standards, as well as the treatment and outcome. More detailed information, such as laboratory tests, was recorded for serious ADRs. Chest images ( $x$ ray or CT) were collected in each case for expert evaluation of respiratory infection; the experts' evaluation was reported back to the investigators for more objective and consistent assessments of the ADRs. ADRs were classified using preferred terms and system organ classes (SOCs) according to the Medical Dictionary for Regulatory Affairs (MedDRA; version 7.1). Infusion reactions were defined as any $\mathrm{ADR}$ occurring during or within $2 \mathrm{~h}$ after the completion of each infusion.

\section{Statistical analysis}

To compare the safety profile of infliximab in this PMS study with the safety profile of on $e^{12}$ of the Japanese clinical trials of infliximab, the rates of ADRs per 100 patient-years and their $95 \%$ confidence intervals (95\% CIs) were calculated. The incidence rates by ADR categories classified by the SOC were computed to provide the ADR pattern.

The risks for bacterial pneumonia were identified by multiple logistic regressions. Serious infusion reactions were analysed by demographics using the Cochran-Armitage test or Fisher's exact test. Cumulative incidence rates of bacterial pneumonia, PCP, tuberculosis and interstitial pneumonitis were plotted after the start of infliximab treatment using the Kaplan-Meier method. P values less than 0.05 were considered significant. Statistical testing was two-sided and performed with SAS version 8.2 (SAS Institute Co. Ltd, Cary, NC).

\section{RESULTS}

\section{Patient demographics}

Table 1 shows the baseline clinical status of 5000 patients consecutively enrolled in the study. Patients were predominantly female, with a mean age of 55.1 years and a mean RA duration of 9.9 years. About $70 \%$ were in RA stages III or IV, and about $90 \%$ were in functional class II or III. Diabetes mellitus and respiratory disease were the most common comorbidities. Patients had an inadequate response to MTX at a mean dose of $7.3 \mathrm{mg} /$ week. Fifty-two patients had been participants in the infliximab clinical trials that were performed several years ago.

\section{Incidence of AEs and ADRs}

AEs developed in 1656 patients and serious AEs were observed in 423 patients. In addition, 1401 patients reported the incidence of any $\mathrm{ADR}$, and 308 patients reported serious ADRs in this PMS study; the incidence rates were $28.0 \%$ and $6.2 \%$, respectively (table 2 ). The incidence rates for total or serious ADRs per 100 patient-years were not significantly different between the current PMS study and the Japanese clinical trial. ${ }^{12}$

Common ADR categories in this PMS study were infections, skin and subcutaneous disorders and systemic disorders. The most common serious categories were infections and respiratory disorders, which were also common in the clinical trial.

\section{Important ADRs}

Since bacterial pneumonia, PCP, tuberculosis, interstitial pneumonitis and serious infusion reactions were common and serious, these were regarded as important ADRs. Table 3 shows the incidence of these ADRs by the five groups of 1000 patients in registration number order.

\section{Bacterial pneumonia}

Bacterial pneumonia developed in 108 cases (2.2\%). The mean age of these patients was 63.5 years (40-79 years), and none was younger than 40 years of age. Bacterial pneumonia occurred after a mean of 2.7 infusions of infliximab.

\section{PCP}

PCP developed in $22(0.4 \%)$ patients. The mean age of these patients was 64.0 years (50-80 years). The pneumonia occurred after a mean of 2.8 infusions of infliximab. The diagnosis of PCP requires microscopic examination in order to identify the organism from a clinical source because the organism cannot be cultured. ${ }^{13}$ Of the 22 patients, polymerase chain reaction (PCR) testing of sputum or bronchoalveolar lavage fluid was positive in 21 cases, and the remaining case was diagnosed clinically by the investigator. Since the organism was not identified in any of these patients, we tentatively categorised them as "suspected" PCP.

\section{Tuberculosis}

Tuberculosis developed in 14 patients $(0.3 \%)$, in whom the organism was identified by culture, smear or PCR. The mean age was 66.1 years (43-76 years). All the cases were graded as serious. Extra-pulmonary tuberculosis was observed in 7 cases (50.0\%). Tuberculosis occurred after a mean of 3.4 infusions of infliximab.

Eleven cases developed in the first 2000 patients (Reg. No. 12,000 ), and 3 cases developed in the last 3000 patients (Reg. No. $2,001-5,000)$. The incidence of tuberculosis among the last 3000 patients was lower than that among the first 2000 patients. The frequency of prophylactic treatment for tuberculosis increased in the latter group (mean rate of $14.0 \%$ in the first 2000 and $22.3 \%$ in the last 3000 patients). 
Table 1 Demographics of the 5000 patients

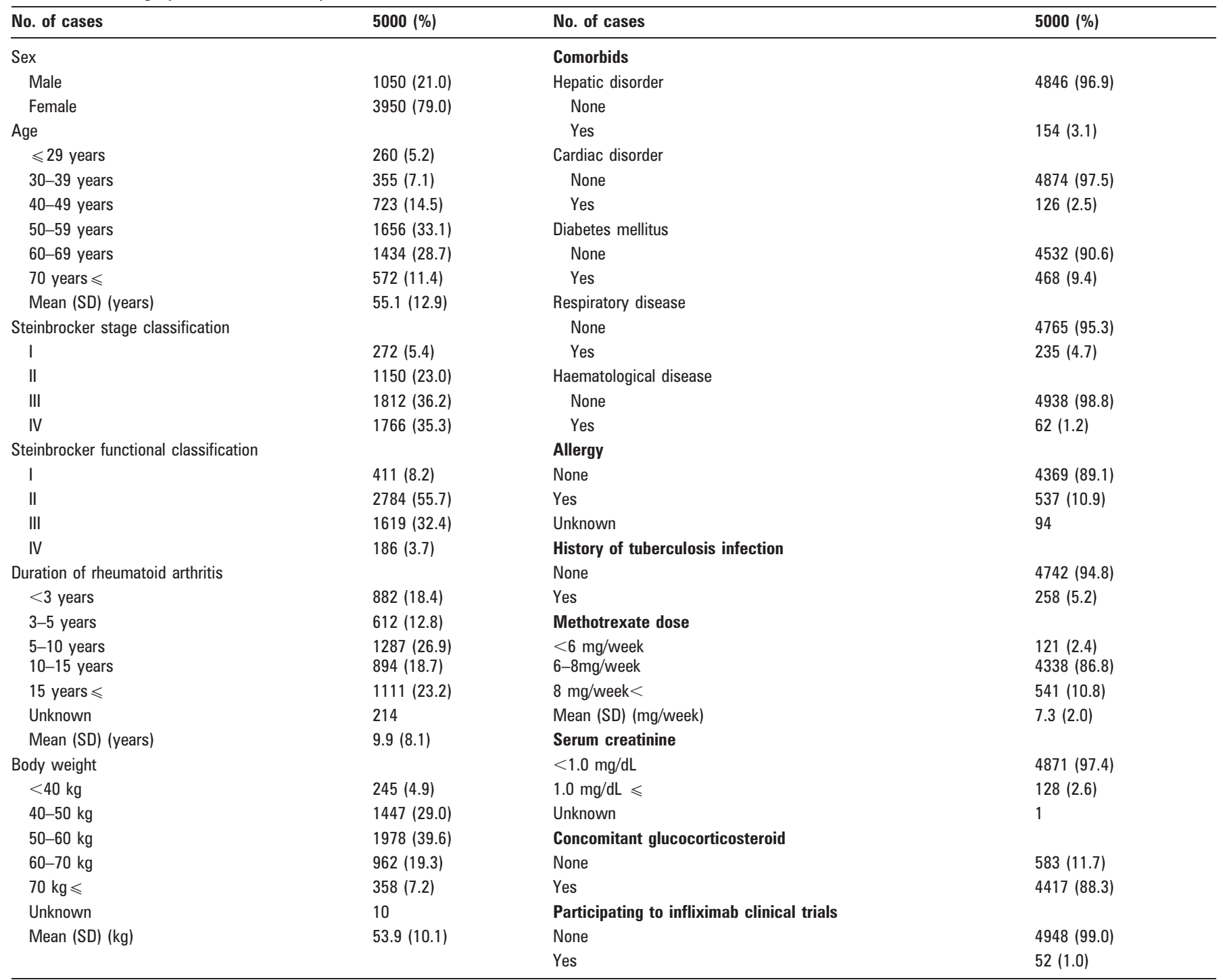

\section{Interstitial pneumonitis}

Interstitial pneumonitis developed in 25 cases (0.5\%). The mean age of these patients was 62.9 years ( $40-77$ years). The pneumonitis occurred after a mean of 2.8 infusions of infliximab.

\section{Infusion reactions}

Infusion reactions occurred in 484 (9.7\%) of the 5000 patients; however, most were not serious and included fever (118 cases, $2.4 \%$ ), hot flushes (78 cases, $1.6 \%$ ) and rash (77 cases, $1.5 \%$ ). Serious infusion reactions developed in 24 cases (0.5\%), with blood pressure decreases ( 9 cases) and anaphylactic/anaphylactoid symptoms (9 cases) occurring most often. Infusion reactions developed in 224 of the first 2000 patients and in 260 of the last 3000 patients. Demographic analysis showed that serious infusion reactions were more commonly observed in participants of the previous clinical trial of infliximab or in patients using $>8 \mathrm{mg} /$ week of MTX $(p<0.001, p=0.004$, respectively).

\section{Risks of bacterial pneumonia}

The risk factors for bacterial pneumonia identified by multiple logistic regression (table 4) were male gender, age 60-70 years or older, Steinbrocker stages III or IV, and comorbid respiratory disease.

\section{Time to occurrence of important ADRs}

The mean number of days from the first infusion to the occurrence of tuberculosis, PCP, bacterial pneumonia and interstitial pneumonitis was 103.1 days (50-184 days), 70.0 days (14-168 days), 72.1 days (2-190 days), and 76.8 days (36-153 days), respectively. Cumulative rates of these ADRs plotted using the Kaplan-Meier method showed that bacterial pneumonia began to develop immediately after the start of the treatment, while tuberculosis, PCP and interstitial pneumonitis began to develop about 1 month after treatment initiation (fig 1).

\section{DISCUSSION}

In clinical trials, the incidence of reported adverse events is accurate, as all adverse events are recorded. However, these trials include strict inclusion and exclusion criteria that deviate from daily clinical practice. Conversely, underestimation can be a significant factor in PMS studies due to under-reporting or lack of reporting, particularly for milder adverse reactions. In this respect, the present study is unique for infliximab, since it is 
Table 2 Incidence of ADRs, serious ADRs and ADRs by the SOC classification

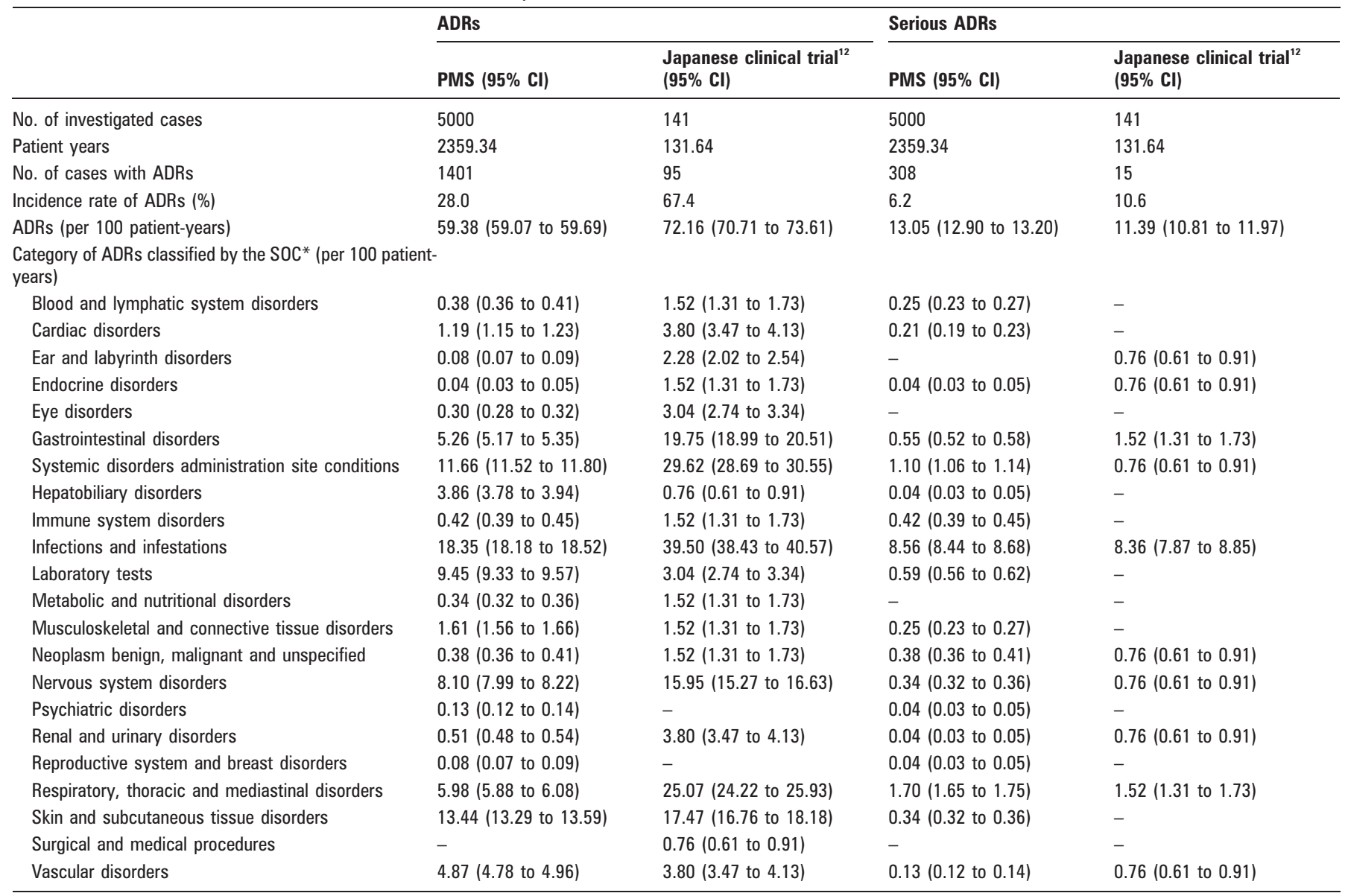

*ADRs were classified using the system organ class (SOC) according to the Medical Dictionary for Regulatory Affairs (MedDRA; version 7.1).

based on a PMS study, but all patients who were to receive infliximab were required to be registered, and all AEs were monitored for 6 months after treatment initiation.

Patients in the PMS study received low-dose MTX (mean: $7.3 \mathrm{mg} /$ week) compared with that in the ATTRACT study ${ }^{2}$ (mean: $16 \mathrm{mg} /$ week). Conversely, the rate of glucocorticosteroid users was higher (88.3\%) than that of the ATTRACT study ${ }^{2}$ (63\%). In addition, $71.6 \%$ patients had high Steinbrocker stage classifications, such as stage III and IV. These clinical features may reflect the unique Japanese situation where the maximum approved dose of MTX ( $8 \mathrm{mg} /$ week) is lower than that in the
US and in European countries. Also, MTX was approved for the treatment of RA in Japan in 1999, indicating that patient exposure to MTX was at most 4-5 years at the PMS study entry. This situation may account for the higher use of glucocorticosteroids and the advanced joint destruction seen in the RA patients in this study. The short duration of followup, 6 months, should also be taken into consideration. Thus, it should be noted that the results obtained in this study have several limitations and are difficult to generalise. Nevertheless, the information may be useful for understanding the safety profile of infliximab in the real world.

Table 3 Important ADRs and overtime

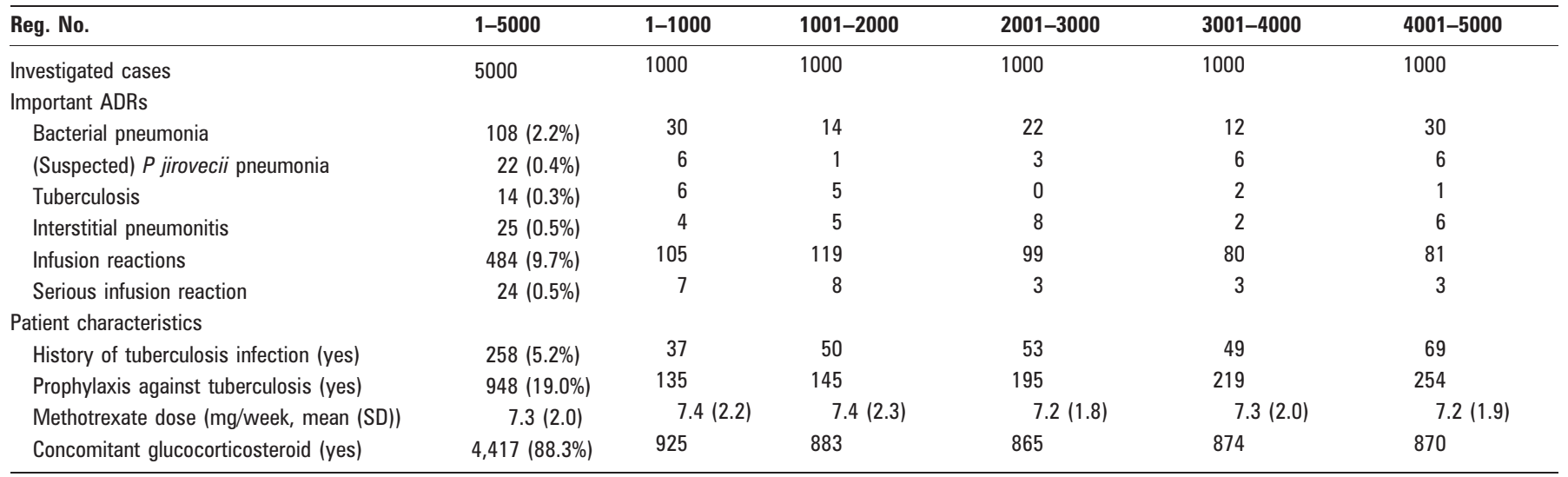


Table 4 Risk factors for bacterial pneumonia

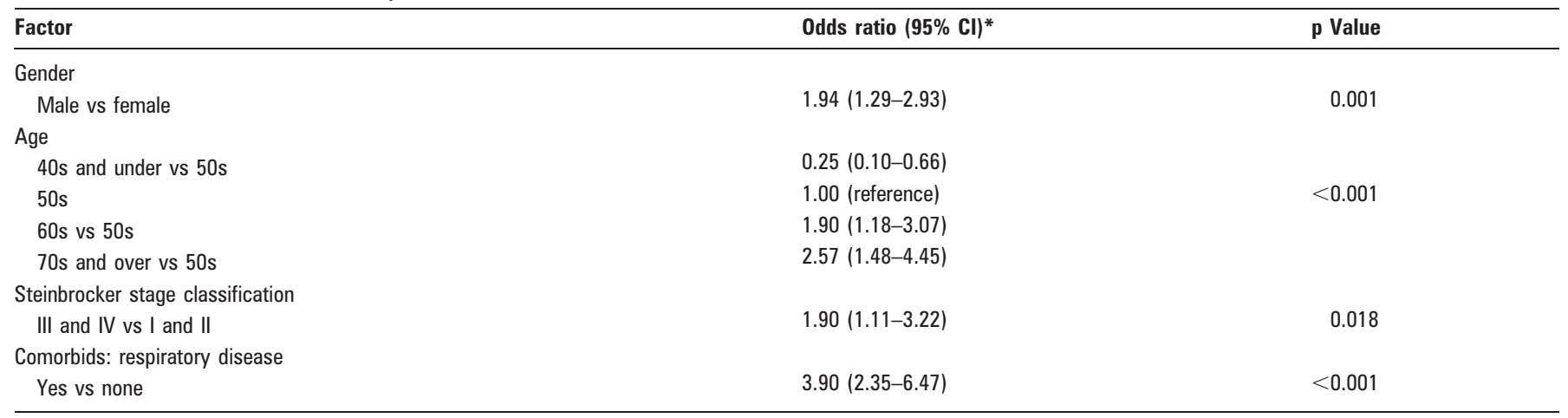

${ }^{*} 95 \% \mathrm{Cl}$, $95 \%$ confidence interval.

The incidence of total ADRs was $28.0 \%$ and the incidence of serious $A D R s$ was $6.2 \%$, with no significant difference observed when compared with the Japanese clinical trial. ${ }^{12}$ The category of ADRs in the PMS study was also similar to that of the clinical trial. ${ }^{12}$ The most common serious ADR was infections, in which bacterial pneumonia was observed most frequently. The incidence rate was $2.0 \%$, which was comparable with that of the ASPIRE study ${ }^{3}(2.2 \%)$, the RABBIT, a cohort study for patients with RA in Germany ${ }^{14}(2.3 \%)$ and the START trial ${ }^{15}$ (serious; $0.8 \%$ ). Wolfe et al investigated the risks of pneumonia hospitalisation in the National Data Bank for Rheumatic Diseases and reported that age, prednisolone use, complications of diabetes or pulmonary disease, cardiovascular disease and $\mathrm{HAO}$ increased the risk of pneumonia. ${ }^{16}$ Our investigation showed that male gender, age, Steinbrocker stage, and comorbid respiratory disease were strong predictors of bacterial pneumonia. The common predictors for pneumonia in these investigations were age and comorbidity of respiratory disease. Although the comorbidity of diabetes mellitus was not a significant risk for the development of pneumonia in our multiple logistic analysis because it was associated with the age and gender, it was identified as a risk in the demographic analysis (data not shown).

Tuberculosis was observed in 14 patients in the present study, of which 11 cases were registered among the first 2000 patients, and the remaining 3 among the last 3000 patients. When the 6 month evaluation of the first 2000 cases was completed, the tuberculosis screening results of the 11 tuberculosis patients were retrospectively reviewed by the expert physicians, and it was discovered that 10 cases had been positive in the screening tests. Although screening for tuberculosis was carried out before the infliximab treatment, some of the investigators did not fully understand the usefulness of the screening, as BCG vaccination had been carried out widely in Japan, and they did not provide prophylactic antituberculosis drugs at the start of the study. After the experts' review, it was decided that tuberculosis screening before infliximab treatment and prophylactic antituberculosis treatment in the case of a suspected past history of tuberculosis should be performed for subsequent patients. As a consequence, prophylactic administration of antituberculosis drugs was increased, and the number of cases of tuberculosis that developed among the last 3000 patients decreased. We continue to strongly recommend that physicians carry out tuberculosis screening tests and begin prophylactic antituberculosis drug use before starting infliximab therapy.

PCP was observed in 22 cases in the present study, but none were reported in the ATTRACT ${ }^{17}$ or $\mathrm{ASPIRE}^{3}$ studies. The organism was not identified microscopically in any cases in the present study, but 21 cases were positive by PCR (PCR was not performed in the remaining case). Saito et a $l^{18}$ evaluated the detectability of $P$ jiroveci DNA in induced sputum of patients with connective tissue diseases and clinical symptoms suggestive of PCP in comparison with that by microscopic examination, and reported the high sensitivity of the PCR method for
Figure 1 Cumulative incidence rate (bacterial pneumonia, interstitial pneumonitis, PCP, tuberculosis).

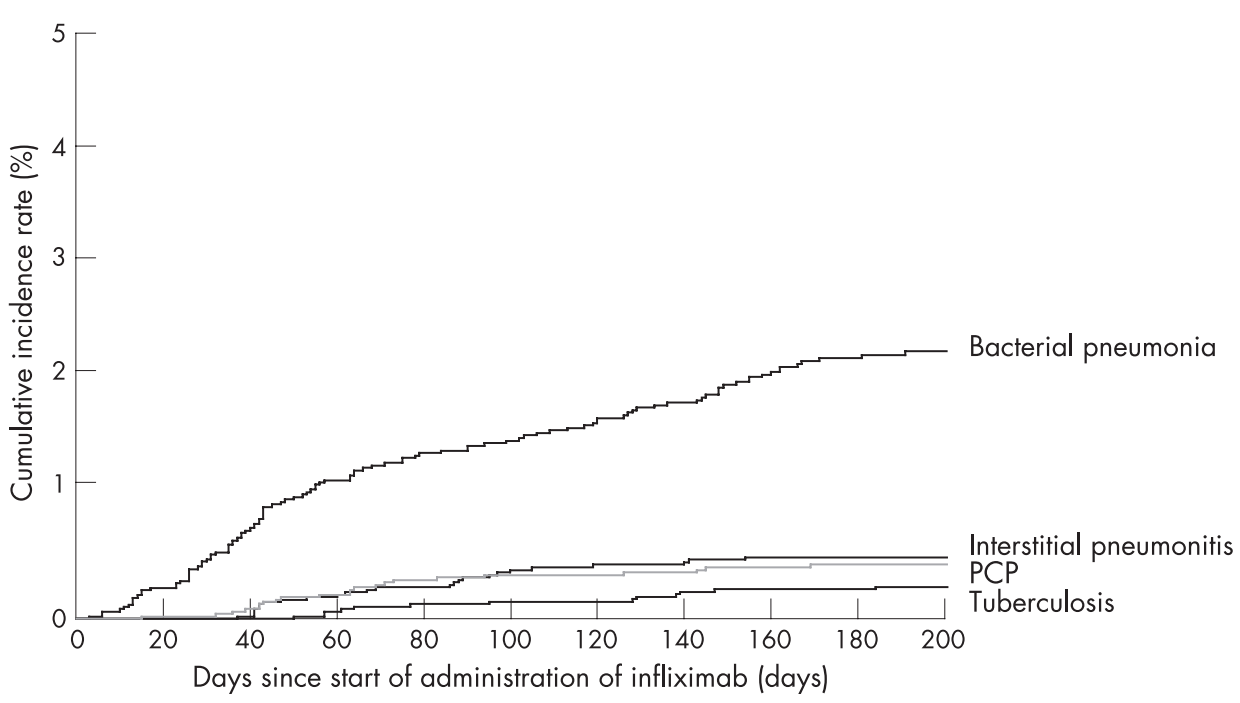


the diagnosis of PCP. The high incidence rate of PCP in the present study may be due to the fact that the diagnosis was based on the very sensitive method.

The bacterial pneumonia cases began to develop immediately after the start of infliximab treatment, whereas tuberculosis, PCP and interstitial pneumonitis began to develop about 1 month later. This difference was considered to be due to the fact that the mitotic rates of the causative organisms in these diseases were different.

A decreasing trend in the incidence rate of infusion reactions was observed among the last 3000 patients, compared with the first 2000 patients. This may be due to the prevailing protocol for managing infusion reactions with the prophylactic use of acetaminophen and antihistamine, and an adjustment of the infliximab infusion speed, as was originally performed in the US. ${ }^{19}$ The serious infusion reactions developed more often in patients who had participated in previous clinical trials of infliximab than in patients who had not. Further investigation is required to establish why the incidence rates were different among those who had participated in previous clinical trials of infliximab.

Acknowledgements: We wish to thank the investigators for the contributions to the conduct of this study, and we thank Dr K. Gilmer (Centocor, Tokyo) for assistance in preparation of the manuscript.

Funding: This study was sponsored by Tanabe Seiyaku Co., Ltd.

Competing interests: YT and YN are full-time employees of Tanabe Seiyaku Co., Ltd. HK has received consulting fees from the sponsor of this study as a consultant.

\section{REFERENCES}

1. Scallon BJ, Moore AM, Trinh H, Knight DM, Ghrayeb J. Chimeric anti-TNF-alpha monoclonal antibody cA2 binds recombinant transmembrane TNF-alpha and activates immune effector functions. Cytokine 1995; 7:251-9.

2. Lipsky PE, van der Heijde DM, St Clair EW, Furst DE, Breedveld FC, Kalden JR, et al. Infliximab and methotrexate in the treatment of rheumatoid arthritis. $N$ Engl J Med 2000;343:1594-602.

3. St Clair EW, van der Heijde DM, Smolen JS, Maini RN, Bathon JM, Emery P, et al. Combination of infliximab and methotrexate therapy for early rheumatoid arthritis. Arthritis Rheum 2004:50:3432-43.

4. van der Heijde DM, Dijkmans B, Geusens P, Sieper J, DeWoody K, Williamson P, et al. Efficacy and safety of infliximab in patients with ankylosing spondylitis. Results of a randomized, placebo-controlled trial (ASSERT). Arthritis Rheum 2005:52:582-91.

5. Antoni CE, Kavanaugh A, Kirkham B, Tutuncu Z, Burmester GR, Schneider U, et al. Sustained benefits of infliximab therapy for dermatologic and articular manifestations of psoriatic arthritis. Results from the infliximab multinational psoriatic arthritis controlled trial (IMPACT). Arthritis Rheum 2005;52:1227-36.

6. Hanauer SB, Feagan BG, Lichtenstein GR, Mayer LF, Schreiber S, Colomel JF, et al. Maintenance infliximab for Crohn's disease: the ACCENT I randomized trial. Lancet 2002;359:1541-9

7. Rutgeerts $\mathbf{P}$, Sandborn WJ, Feagan BG, Reinisch W, Olson A, Johanns J, et al. Infliximab for induction and maintenance therapy for ulcerative colitis. N Engl J Med 2005;353:2462-76

8. Gottlieb AB, Evans R, Li S, Dooley LT, Guzzo CA, Baker D, et al. Infliximab induction therapy for patients with severe plaque-type psoriasis: A randomized, double-blind, placebo-controlled trial. J Am Acad Dermatol 2004;49:534-42.

9. Goekoop-Ruiterman YPM, de Vries-Bouwstra JK, Allaart CF, van Zeben D, Kerstens PJSM, Hazes JMW, et al. Clinical and radiographic outcomes of fou different treatment strategies in patients with early rheumatoid arthritis (the BeSt study). A randomized, controlled trial. Arthritis Rheum 2005;52:3381-90.

10. Keane J, Gershon S, Wise RP, Mirabile-Levens E, Kasznica J, Schwieterman WD, et al. Tuberculosis associated with infliximab, a tumor necrosis factor a-neutralizing agent. N Engl J Med 2001;345:1098-104.

11. Miyasaka M, Takeuchi T, Eguchi K. Proposed Japanese guidelines for the use of infliximab for rheumatoid arthritis [Erratum: Mod Rheumatol 2005;15:322]. Mod Rheumatol 2005;15:4-8.

12. Abe T, Takeuchi $T$, Miyasaka N, Hashimoto $H$, Kondo $H$, Ichikawa $Y$, et al. A multicenter, double-blind, randomized, placebo controlled trial of infliximab combined with low dose methotrexate in Japanese patients with rheumatoid arthritis. J Rheumatol 2006;33:37-44

13. Sing A, Trebesius K, Roggenkamp A, Russmann H, Tybus K, Pfaff F, et al. Evaluation of diagnostic value and epidemiological implications of PCR for Pneumocystis carinii in different immunosuppressed and immunocompetent patient groups. J Clin Microbiol 2000;38:1461-7.

14. Listing J, Strangfeld A, Kary S, Rau R, Ulrich von Hinueber, Stoyanova-Scholz M, et al. Infections in patients with rheumatoid arthritis treated with biologic agents. Arthritis Rheum 2005:52:3403-12.

15. Westhovens R, Yocum D, Han J, Berman A, Strusberg I, Geusens P, et al. The safety of infliximab, combined with background treatments, among patients with rheumatoid arthritis and various comorbidities: a large, randomized, placebocontrolled trial. Arthritis Rheum 2006:54:1075-86.

16. Wolfe F, Caplan L, Michaud K. Treatment for rheumatoid arthritis and the risk of hospitalization for pneumonia. Associations with prednisone, disease-modifying antirheumatic drugs, and anti-tumor necrosis factor therapy. Arthritis Rheum 2006;54:628-34.

17. Maini RN, Breedveld FC, Kalden JR, Smolen JS, Furst D, Weisman MH, et al. Sustained improvement over two years in physical function, structural damage, and signs and symptoms among patients with rheumatoid arthritis treated with infliximab and methotrexate. Arthritis Rheum 2004:50:1051-65.

18. Saito K, Nakayamada S, Nakano K, Tokunaga M, Tsujimura S, Nakatsuka K, et al Detection of Pneumocystis carinni by DNA amplification in patients with connective tissue disease: re-evaluation of clinical features of $P$. carinni pneumonia in rheumatic diseases. Rheumatology (Oxford) 2004;43:479-85.

19. Cheifetz A, Smedley M, Martin S, Reiter M, Leone G, Mayer L, et al. The incidence and management of infusion reactions to infliximab: a large center experience. Am J Gastroenterol 2003;98:1315-24. 